\title{
The Present Situations, Causes, and Clinical Therapy of Depression in College Students
}

\begin{abstract}
Yitong Wang
The University of Warwick/ psychology and education/CV4 7AL

Abstract. The incidence of depression in college students is increasing in recent years, which could lead to physical and mental diseases and negative academic outcomes. The causes of depression are related to both genetic and environmental factors. The main clinical therapy approaches of depression are medication and psychotherapy. In addition to clinical treatment, supports from educators are essential to students for intervention and prevention of depression in a school setting. Promoting students' awareness of depression and abilities of stress management is beneficial for their mental health and academic outcomes.
\end{abstract}

Keywords: depression, college students, treatment

\section{Introduction}

Mental health problems have been concerned as a large component in the Global Burden Disease (GBD) which generated by the World Health Organization (WHO) provides comprehensive information in terms of disease and injury. Depression has been classified as one of the most prevalent mental disorders which is estimated to affect 340 million people in the globe, and is considered to be the most primary cause of disease burden by 2020 , although it was the fourth main cause in $2000^{[1,2]}$. The prevalence of depression is increasing among university students who are in their vital period of life, experiencing transition from youths to adults. The study conducted by Chen et al. ${ }^{[3]}$ indicates that moderate depression is prevalent among Chinese university students, and there are 4 percent of Chinese university students are suffering major depression. Depression would lead to negative results on campus life among this special group, like poor academic outcomes and social problems. In addition, depression may cause physical impairments, including sleep disorders, loss of appetite, and even high tendency of suicide. Furthermore, this study also points out that many lifelong mental diseases begin at university period. Therefore, studies for depression among undergraduate students are significant for educators to help students with their mental health and campus experiences. The significance of studies for depression has been noticed by worldwide professionals and researchers in this filed, and research in various facets of depressive disorder is being developed and improved. Firstly, as depression is a chronic illness which may last the whole lifespan, current studies on depression consider the factor that patients are from different age groups, and divide subjects into children, adolescents, adults, and older people ${ }^{[4][5]}$. In addition, other psychiatric problems and physical illnesses associated with depression and their relationship with depression are also included in the scope of depression research, like anxiety, attention deficit hyperactivity disorder (ADHD), and cardio-cerebrovascular disease. Zhang [6] argues that depression often co-occur with these disorders, and the relationship between depression and these disorders may be reciprocal causation. Moreover, there are quite a number of current studies focus on the causes and influencing factors of depression which could be roughly divided into genetic factor and environmental factor. The present studies reach an agreement on genetic factor that the incidence of depression has high level of familial aggregation and affected by polygenic inheritance ${ }^{[6]}$. Finally, current studies are also focus on seeking effective treatment measures and interventions of depression, which could directly offer benefits to patients.

In this paper, I will discuss the depression among university students from the perspective of diagnosis, scale, causes and clinical therapy.

\section{Diagnosis and scale of depression}

\subsection{Diagnosis and symptoms}

One of the most common used diagnostic criteria for depression is pointed out in the DSM-IV which is published by American Psychiatric Association. The criteria outlines two main symptoms (depressed mood and loss of pleasure and interest) and other performances, involving (1) slow thought and decreased physical activity that could be observed by others, (2) obvious reduction of weight without diet and abnormal variety in appetite, (3) feeling of tired and lack of energy, (4) feeling of worthless, (5) disability of concentrating (6) and recurrent thought of death and suicide. A person who can be determined as depression need to meet five or more above conditions every day during a 2-week period which at least involve one main symptom. Another assessment used wildly for diagnosis of depression is from the tenth edition of International Classification of Diseases (ICD-10). Similarly, this criteria put forward following symptoms: (1) depressed mood, (2) anhedonia, (3) loss of vigor, (4) decline of attention, (5) thought about worthless and guilty, (6) pessimism and hopelessness, (7) tendency for suicide and self-harm behaviours, (8) loss of self-confidence, (9) sleep disorder, (10) reduction of appetite. If individual presents 4 or more of these conditions which include more than 2 symptoms from the first three conditions, and these 
performances last more than 2 weeks, the depressed disorder could be diagnosed ${ }^{[7]}$.

In the university setting, students with depression may behave following symptoms: (a) as this special group of students usually show low moods and unable to feel enjoyments in daily activities, they lose interests in academic courses and barely participate extracurricular activities; (b) as a result, depressed students' interpersonal relationships with peers and teachers would be negatively affected, and some of them may addicted to computer games because lack of social communication; (c) another symptom of depressed students is attention deficit that they unable to focus on class and other academic tasks, which lead to poor academic outcomes; (d) depressed students always have irrational cognition about themselves that they do not have capacities to do anything well and feel hopeless about their life (e) dull performance is also a typical symptom of students suffering depression with slow thoughts and actions; (f) moreover, depression could also results in several physical symptoms, such as headache, insomnia, and digestive problems ${ }^{[8]}$.

\subsection{Scale of depression}

A variety of scales for depression have been widely applied in related research, which is a method of screening for depression with descriptive language to determine the level of depression in a time period. Compared to diagnosis of depression, scores of a scale for depression could reflect individual's depressive behaviours during a time period and indicate whether this person should seek a mental health professional for further diagnosis.

The Hamilton Depression Rating Scale (HAM-D) has been demonstrated effective for depression evaluation, which need to be completed by professional with clinical experiences. The HAM-D which has 17 items takes clinician about 15-20 minutes to be completed. There are 8 items from the scale are rated by 5-point scale from 0 to 4 and 9 items are rated by 3 -point scale from 0 to 2 . The total scores represent the level of depression. A total score of 0-7 is considered as an acceptable range. However, an individual with total score above 20 is considered to be moderate depression and needs clinical treatment ${ }^{[9]}$.

Another kind of measurement is self-report scale that be evaluated by patients. Compared with the limitation of clinician-administered scales that it may cause heavy work burden to professionals and insufficiency of staff, selfreport scale is an effective and reliable tool, and it could gain more accurate information about patient's internal mental situation. Beck Depression Inventory (BDI) is considered consist with the DSM diagnostic criteria. The questionnaire is consist of 21 items and takes patients about 5 to 10 minutes fill out. Each item include 4 choices of descriptive statements which represent different severity of depression. The total score range is from 0 to 63 , and the operation manual gives the meaning of score: total score from 0 to 13 is normal, from 14 to 19 indicates mild depression, from 20 to 28 indicates moderate depression, and from 29 to 63 indicates severe depression ${ }^{[10]}$.
Moreover, the Patient Health Questionnaire (PHQ-9) is also a valid self-administered scale for recognition of depression and inspection of treatment, which is based on the DSM-IV criteria of depressive disorder. The questionnaire includes 9 items with 4-point Likert scoring scale from 0 (not at all) to 3 (nearly every day), asking patients to fill out according to their frequency of these depressive symptoms over the past 2 -week period. The meaning of total score has been given: $0-4$ is no depression, 5-9 is mild depression, 10-14 is moderate depression, $15-19$ is moderately severe depression, and 20-27 is severe depression ${ }^{[11]}$.

\section{CAUSES OF DEPRESSION}

\subsection{Familial Aggregation}

The results from several family studies with controlled methods explain that depression is a kind of familiar illness. Compared to comparison subjects, patients with depression appear familial aggregation, and relationships between depression in patients and depression in their first-degree relatives are highly correlated. However, this influence of family members on patients could not be determined whether it is caused by genetic factors or environmental factors because of the relative invariance of a family's environment ${ }^{[12]}$.

\subsection{Genetic factor}

A number of previous works indicate that depression which is hereditary is highly related to genic elements. It is pretty efficient to utilize twins in inherent problems. Kendler and Prescott ${ }^{[13]}$ conducted a large scale twins experiment that recruited 3790 pairs of twins including male-male twins, female-female-twins, and male-female twins. The study gained information by telephone interview and got results that the effect of heredity contributes around $39 \%$ to the possibility of suffering major depression, and the rest $61 \%$ is caused by environmental factor. In addition, there is no significant difference in genetic risk between males and females, but the genetic factor may have different influences on the risk of depression between male group and female group. Similarly, the research designed by Lyons et al. ${ }^{[14]}$ collected information from 3372 pairs of male twins from Vietnam, comparing to the study which included only female participants. The results show that the heritability of depression is equal for males and females, and genetic elements may have more powerful influence on the higher level of depression while less severe depression is more effected by environmental factors.

Recently, Howard et al. ${ }^{[15]}$ gave more specific findings about the effect of genetic factors on depression in their study. The study collected a large amount of data including 807553 individuals to meta-analyze and concluded that some genes and gene-pathways which are linked with neurotransmission and synaptic structure are related to 
depression, including 102 independent variants, 269 genes, and 15 gene-sets. The results from this research determined the significance of prefrontal cortex in the brain and provided benefit for therapy of targeted medicine for depression.

\subsection{Family environment}

Family environment could influence a person in many facets, like personality, living habits, and physical health, as the function of family is not only offering financial help, but also education, maintenance, and offering emotional support. As a result, the depression and other mental disorders among university students may cause by their parents. The conclusions from study explored by Guan et al. [16] indicate that the support from family is significantly related to depression for males, and negative family events and poor state of economy could lead to depressive disorder. The participants from this study were aged beyond 18 from Chinese rural region. Comparing to city residents, university students from these poor areas are more likely to have low self-esteem and economic stress.

In addition, the parenting style from parents could also be a risk factor for individual's depression. Applying a structural equation modeling, Kim and $\mathrm{Ge}{ }^{[17]}$ examined the relationship between the depressive symptoms of adolescents and parenting practices, involving inductive reasoning, monitoring, and harsh discipline. The authors noted that the level of adolescent depression is negatively related to the perception of inductive reasoning and parental monitoring, and higher level of harsh discipline from parents may lead to higher level of adolescent depression. Youths are likely to feel too much pressure and deficiency of freedom in the excessive restrictive family environments, and they may continue this negative mental states to college.

\subsection{Interpersonal relationship}

As college is an inclusive environment that people from different regions, various culture flock together, some students would encounter problems about interpersonal relationships, and the aggravation of this sort of disturbance may result in mental problems. Firstly, interpersonal conflict is a common social problem among college students which could bring negative emotions and mental stress for individuals. These contradictions may caused by differences on perception and insufficiency of understanding. What's more, some students would suffer discriminated against because of their economic situation or some deficiencies. Secondly, some depressive students report that they lack of friends in the campus life, so these people could not shire their emotions to others and lack of social supports without friends. Furthermore, the inappropriate handling and strike of romantic relationships may results in extreme thoughts among young adults ${ }^{[18]}$. Finally, Vidyanidhi and Sudhir ${ }^{[19]}$ point out that clinical depression patients have higher level of interpersonal sensitivity than comparison group, demonstrating the theory that interpersonal sensitivity is a typical personality prone to depression.

\subsection{Personality and adaption}

Extensive studies show the evidences that personality has influence on individual's depression. Firstly, Dyson and Renk ${ }^{[20]}$ report that the degree of adaption of freshmen could significantly predict their level of depression. Entrancing into college and adapting new environments may be stressful experiences for some freshmen, because they need to face some challenges like independent living without parents, establishment of new interpersonal circle, and developing academic abilities for advanced curriculum. So under these circumstances, in order to have making good adaption, students may need to alert their strategies, coping with problems and challenges, and adjusting state of mind. Otherwise, they may have depressive symptoms because of maladjustments.

Secondly, the perception of stress is related to students' depression. Besides academic and social stress, university students also need to face pressures of employment that the number of college graduates increases every year and be estimated to reach 874 million by 2020 in China. Therefore, coping with and manage various stress is crucial for college students' mental health.

In addition, Starley ${ }^{[21]}$ mentioned a "roots and leaves" model that perfectionism is likened as the root of a plant and many types of mental health disorders are likened as leaves of the plant which include depression. Students with high level of perfectionism may have unrealistic exceptions for themselves and worry about making mistakes all the time, which is a strong tendency for depression.

\section{TREATMENT OF DEPRESSION}

With the growing prevalence and severity of depression, there have been a number of methods on therapy and management of depression from different aspects, like pharmacologic therapy, neurological therapy, psychological treatment, excise treatment, and music treatment. In the meantime, for the current situation of depression among university students, educators should provide efforts and effective inventions to assist treatment process.

\subsection{Clinical therapy}

\subsubsection{Depression medicine}

Antidepressant is a common choice for depression therapy which has effects on brains through neurotransmission systems to ease depressive symptoms. Selective serotonin reuptake inhibitors (SSRIs) such as sertraline (Zoloft), citalopram (Celexa), and fluvoxamine (Luvox), is the most widely used depression medicine which has satisfying 
therapeutic results and mild side effects. Although the negative additional effects are slight, some patients would still have several physical symptoms during the period that taking this kind of medicine, like stomach discomfort, nausea, dizziness, debilitation, sleep disorder, and headache. Comparing to this newer depression drug, Tricyclic antidepressants (TCAs) and Monoamine oxidase inhibitors (MAOIs) are two older medications for depression whereas they are thought having relative severe side effects and risk of serious interactions with some food and medicine from today's medical point of view ${ }^{[22]}$.

\subsubsection{Psychotherapy}

Cognitive-behavioural therapy is a kind of psychotherapy which could help patients aware that their negative thoughts and assumptions are unreasonable and alter these irrational views under the assists of therapists with cognitive theories. Another effective method is interpersonal psychotherapy that therapists develop treatment plans for each patient according to their characteristics. The main purpose of this method is helping patients identify and promote interpersonal problems related to their depressive emotions with cooperation between therapists and patients ${ }^{[23]}$.

In the most clinical treatment for depression, psychotherapy is often combined with pharmacotherapy, which could gain better outcomes than using only one method. The random control trail designed by De Jonghe et al. ${ }^{[24]}$ supports the above view. Patients with depression in this study were randomly divided into medicine group and combined therapy group. The results indicate that the combined method is significantly more effective than only using medication that the average success rate of medicine group was $40.7 \%$ and for combined group was 59.2 after 24 weeks. Moreover, combined therapy is more acceptable than pharmacotherapy that patients are more likely to insist treatment process with this approach.

\subsubsection{Physical exercise treatment}

Physical activity as a value supplementary treatment for depression has been demonstrated in various reports, which has effects on improvement of the rest symptoms and prevent of recurrence. The UK National Institute for Health and Clinical Excellence (NICE) suggests that for patients with mild to moderate depression, regular activity under supervision could be applied as a low-intensity method in the follow-up therapy, and the recommended frequency is three times a week (45-60 minutes each time) lasting 10-14 weeks ${ }^{[25]}$. Trivedi et al. ${ }^{[26]}$ conducted a pilot study to investigate the assist effect of exercise treatment for depressive disorder. Patients with depression were asked to participant a 12-week exercise program, and they also continued to have their antidepressants at this period. Their total scores of depression scale significantly decreased after this program, which preliminarily demonstrated that physical activity has positive effects on depression therapy.

\subsection{Educators}

\subsubsection{Promoting personal health of students}

Encouraging students to form healthy living habits is benefit, including appropriate exercises, regular sleeps, and balanced diets. A healthy physical situation is the foundation of a student's study life, which could provide sufficient energy and pleasant mood. In addition, helping students well balance their work and life is a noteworthy point for educators. Educational staff should arrange curriculums carefully that making students have time off to relax themselves from stressful academic tasks. Appropriate rest could help students make adjustments and energetically engage in the following works $^{[27]}$.

\subsubsection{Improving student's understanding of depression and developing psychological support}

An online survey in Chinese reports that most college students lack of knowledge of depression that they merely have shallow understandings of this disease but are ignorant of its symptoms and treatments ${ }^{[28]}$. As depression is a prevalent disorder among college student, popularizing knowledge of this field is valued. Educators should conduct lectures and activities to help students learn more information about depression. As a result, students could aware their negative emotion status in the early time and seek professional assists promptly. In addition, developing the construction of psychological consulting room in school is beneficial for university students. Students could talk to psychological consultant and gain professional suggestion when they feel stress out or unmanageable depressive moods, and the stranger character of psychological consultant could solve the problem that some students would feel embarrassed to tell their troubles to familiar people ${ }^{[18]}$.

\subsubsection{Creating a positive learning environment}

Providing appropriate autonomous right to students is beneficial for both students and educators, including giving opinion about curriculums, evaluating teaching methods, and summarize the learning effects at regular intervals. Then students would perform more actively in study because the sense of involvement about their learning experiences, in turn administrators could obtain effective suggestions from student's perspective. Moreover, group activities could offer opportunities for students about extending social circle and talking feelings and thoughts to peers, and help students develop empathy ${ }^{[27]}$.

\subsubsection{Promoting the ability of stress management}

Teaching students skills about stress management including being aware of stressful emotions, identifying the source of stress, and coping with anxiety and stress. In addition, 
Sawatzky et al. ${ }^{[29]}$ gave the evidence that self-efficacy has partially mediating effect between stress and depression. According to Bandura's social cognitive theory, self-efficacy is defined as individuals' level of confidence about their abilities to succeeding in a specific context or completing a task, which includes the capacity of stress adjustment ${ }^{[30]}$. So educators could train students to establish the high level of self-efficacy by providing positive feedbacks and appropriate supports, then students would believe that they could have good adjustments and managements about their stress.

\section{CONCLUSION}

The prevalence of depression among Chinese university students is rising in recent years which could lead to negative effects on both psychology and physic. This phenomenon need to be noticed by people from various domains of society especially parents and educators who could timely discover problems and directly offer effective preventions and interventions. So the study on student depression is significant in psychology and education. Further research could explore mediating and moderating relationships between related factors and depression, figuring out the specific mechanism. Moreover, for the existing study results about effective preventions and interventions, further research could expand to different regions and populations. However, appropriate modifications are necessary because of some diversities like cultural differences and age differences.

\section{REFERENCES}

[1] Moussavi, S., et al., Depression, chronic diseases, and decrements in health: results from the World Health Surveys. The Lancet, 2007. 370(9590): p. 851-858.

[2] Greden, J.F., The burden of recurrent depression: causes, consequences, and future prospects. Journal of Clinical Psychiatry, 2001. 62: p. 5-9.

[3] Chen, L., et al., Depression among Chinese university students: prevalence and socio-demographic correlates. PloS one, 2013. 8(3): p. 1932-6203.

[4] Weisz, J.R., C.A. McCarty, and S.M. Valeri, Effects of psychotherapy for depression in children and adolescents: a meta-analysis. Psychological bulletin, 2006. 132(1): p. 1939-1455.

[5] Fiske, A., J.L. Wetherell, and M. Gatz, Depression in older adults. Annual review of clinical psychology, 2009. 5: p. 363-389.

[6] Jun Ling, Z., Research status and influencing factors of depression. China Foreign Medical Treatment, 2008. 25.

[7] Gruenberg, A.M., R.D. Goldstein, and H.A. Pincus, Classification of depression: research and diagnostic criteria: DSM-IV and ICD-10. Biology of Depression, 2005. 11: p. 43.
[8] Jing, W., Discussion on the current situation of depression in college students. Chinese General Practice Nursing, 2013. 11: p. 3419-3420.

[9] Hamilton, M.A.X., Development of a rating scale for primary depressive illness. British journal of social and clinical psychology, 1967. 6(4): p. 278-296.

[10] Jackson-Koku, G., Beck depression inventory. Occupational Medicine, 2016. 66(2): p. 174-175.

[11] Kroenke, K., R.L. Spitzer, and J.B.W. Williams, The PHQ-9: validity of a brief depression severity measure. Journal of general internal medicine, 2001. 16(9): p. 606-613.

[12] Sullivan, P.F., M.C. Neale, and K.S. Kendler, Genetic epidemiology of major depression: review and metaanalysis. American Journal of Psychiatry, 2000. 157(10): p. 1552-1562.

[13] Kendler, K.S. and C.A. Prescott, A population-based twin study of lifetime major depression in men and women. Archives of general psychiatry, 1999. 56(1): p. $39-44$.

[14] 14. Lyons, M.J., et al., A registry-based twin study of depression in men. Archives of general psychiatry, 1998. 55(5): p. 468-472.

[15] Howard, D.M., et al., Genome-wide meta-analysis of depression identifies 102 independent variants and highlights the importance of the prefrontal brain regions. Nature neuroscience, 2019. 22(3): p. 343.

[16] Guan, C., et al., Family Environment and Depression: A Population-Based Analysis of Gender Differences in Rural China. Journal of Family Issues, 2014. 35(4): p. 481-500.

[17] Kim, S.Y. and X. Ge, Parenting practices and adolescent depressive symptoms in Chinese American families. Journal of Family Psychology, 2000. 14(3): p. 420

[18] Ying Chao, M. and G. Hui Fang, Causes, intervention and prevention of depression among college students. Ability And Wisdom, 2018: p. 62-64.

[19] Vidyanidhi, K. and P.M. Sudhir, Interpersonal sensitivity and dysfunctional cognitions in social anxiety and depression. Asian Journal of Psychiatry, 2009. 2(1): p. 25-28.

[20] Dyson, R. and K. Renk, Freshmen adaptation to university life: Depressive symptoms, stress, and coping. Journal of clinical psychology, 2006. 62(10): p. 1231-1244.

[21] Starley, D., Perfectionism: a challenging but worthwhile research area for educational psychology. Educational Psychology in Practice, 2019. 35(2): p. 121-146.

[22] Kristeen, C., What medications help treat depression? 2017. 
[23] Luty, S.E., et al., Randomised controlled trial of interpersonal psychotherapy and cognitivebehavioural therapy for depression. The British Journal of Psychiatry, 2007. 190(6): p. 496-502.

[24] De Jonghe, F., et al., Combining psychotherapy and antidepressants in the treatment of depression. Journal of affective disorders, 2001. 64(2-3): p. 217-229.

[25] Blake, H., Physical activity and exercise in the treatment of depression. Frontiers in Psychiatry, 2012. 3: p. 106

[26] Trivedi, M.H., et al., Exercise as an augmentation strategy for treatment of major depression. Journal of Psychiatric Practice ${ }^{\circledR}, 2006.12(4)$ : p. 205-213.

[27] Dyrbye, L.N., M.R. Thomas, and T.D. Shanafelt. Medical student distress: causes, consequences, and proposed solutions. 2005. Elsevier.

[28] Chao Guang, H., et al., A study on the current situation of depression among college students. PSY, 2018: p. 34-36.

[29] Sawatzky, R.G., et al., Stress and depression in students: the mediating role of stress management selfefficacy. Nursing research, 2012. 61(1): p. 13-21.

[30] Bandura, A. and N.E. Adams, Analysis of self-efficacy theory of behavioral change. Cognitive therapy and research, 1977. 1(4): p. 287-310. 